\title{
Political Waters. Governmental water management and neoliberal reforms in Khartoum/Sudan
}

\author{
L. Crombé \\ rés-EAU P10/WATER network P10, Université Paris Ouest Nanterre La Défense, 200 Av. de la République, \\ 92000 Nanterre, France
}

Université de Fribourg, Unit of Geography - Chemin du Musée 4, 1700 Fribourg, Switzerland

Correspondence to: L. Crombé (laure.crombe@unifr.ch)

Beckedorf, A.-S.: Political Waters. Governmental water management and neoliberal reforms in Khartoum/Sudan, Forum Politische Geographie, Bd. 7, LIT Verlag Berlin, 312 pp., ISBN-13: 978-3-643-90216-0, €34.90, 2012.

Cet ouvrage, issu d'une thèse de doctorat en Géographie (Université de Bayreuth, Allemagne) et de recherches menées au sein du programme de recherche WAMAKHAIR, propose une analyse de la gestion gouvernementale de l'eau dans l'agglomération de Khartoum (Soudan) au regard des réformes néolibérales en cours. Appuyé sur les travaux d'auteurs tels que K. Bakker ou E. Swyngedouw, cet ouvrage illustre comment les réflexions menées en political ecology analysent les récentes évolutions du secteur de l'eau et remettent au centre du questionnement l'importance du politique et des enjeux de pouvoir autour de la gestion de la ressource. Ce livre est composé de 8 chapitres à travers lesquels la mise en perspective de deux champs conceptuels, la political ecology et la sociologie des organisations (chapitre 1), appuyée sur un impressionnant travail empirique (chapitres 3 à 7), permet de comprendre la mise en œuvre des récentes réformes néolibérales à travers l'étude des restructurations internes de la Khartoum State Water Corporation (KSWC) et de ces acteurs, et au-delà d'éclairer le fonctionnement de systémes complexes, ici ceux du régime et de la société soudanais.

A.-S. Beckedorf se positionne ici au niveau institutionnel dans l'étude de la gestion de l'eau. Elle s'appuie notamment sur les travaux de Karen Bakker, pour analyser ces réformes dans le cadre soudanais. Trois des principaux concepts du néolibéralisme ont été fortement promus par le gouvernement soudanais depuis les années 1990, la décentralisation, la privatisation et la commercialisation. L'auteure mobilise, dans un premier temps, deux aspects de la political ecology, comme cadre d'analyse des transformations socio-environnementales. Tout d'abord, la prise en compte de la "matérialité" de l'eau ("water's specific constraints as a physical resource" p. 18), améne, en contre-pied des études d'économie politique, à repenser la gestion de l'eau, les principes de régulation, le dualisme public et privé, sur la base des interactions entre caractéristiques physiques de l'eau et aspects socio-économiques. Dans un deuxiéme temps, A.S. Beckedorf souligne l'importance des relations de pouvoir et du politique dans les interactions sociétés-nature. Cet aspect est d'ailleurs le plus exploité dans ce travail et améne à reconsidérer le rôle de l'État dans la gestion de l'eau ainsi que son influence dans la mise en place des réformes néolibérales. Ainsi, aprés un chapitre 3 de mise en contexte historico-politique de la gestion de l'eau au Soudan et à Khartoum, l'argumentation est construite autour des trois principaux chapitres empiriques qui présentent la mise en œuvre des réformes de décentralisation (chapitre 4), privatisation (chapitre 5) et commercialisation (chapitre 6) respectivement mises en paralléle avec les notions de pouvoir ("power"), d'état ("state") et de politique ("politics"). Une seconde trame conceptuelle autour de la sociologie des organisations, est alors mobilisée pour l'analyse interne et micro-locale de la KSWC. Cette approche reste néanmoins secondaire d'un point de vue théorique. Le recourt à différents auteurs, Giddens, Neuberger et leur théories, peut ne sembler, dans un premier temps, qu'un outil de justification méthodologique dans le cadre d'un exercice de doctorat. Cependant, derriére une maitrise de ces approches sociologiques qui semble plus superficielle, il convient de souligner que c'est dans ce croisement, entre la political ecology et cette "micropolitique des organisations", que l'apport, de cet ouvrage pour la géographie, réside.

C'est donc à travers ce double cadre qu'A. S. Beckedorf décrit la mise en place des réformes néolibérales depuis les 
années 1990 et les logiques qui les sous-tendent. L'auteure montre que l'imbrication des différentes logiques individuelles, morales, politiques aménent à des résultats contrastés, une variabilité dans l'application et la contestation de ces principes au sein de l'organisation gouvernementale mais aussi, à d'autres niveaux, du régime et de la société. Enfin elle met en lumiére l'imbrication forte des processus qui régissent une politique sectorielle locale avec le fonctionnement général du régime soudanais. L'étude des processus de décentralisation/recentralisation (chapitre 4) démontre que des enjeux politiques concurrencent ceux d'ordre technique. Le constant décalage entre la promotion de la décentralisation dans le secteur de l'eau et son imparfaite mise en œuvre témoigne d'une certaine réticence à la délégation du pouvoir de la part des dirigeants khartoumois et soudanais tout comme d'une contestation de cette réforme à travers des politiques de recentralisation qui permettent au gouvernement central de "diviser pour mieux régner". Il apparait donc comme un moyen d'une part, de servir les intérêts personnels des dirigeants de ce secteur, et d'autre part, pour le régime de 1989 d'asseoir son pouvoir. Dans un second temps (chapitre 5), l'auteure montre comment cette privatisation est avant tout une affaire d'état, largement "gouvernementalisée" et orchestrée par et à l'intérieur des institutions du régime. Cette hybridation du public et du privé est qualifiée ici de "government-private continuum". Cette notion permet d'appréhender combien ces deux sphéres sont imbriquées l'une dans l'autre et leur place dans la gestion de l'eau renégociée au gré des dynamiques sociopolitiques. Enfin (chapitre 6) les processus de commercialisation (reconfiguration interne de la KSWC, la réforme de la tarification de l'eau et le développement d'une gestion intégrée de l'eau) sont analysés sous le prisme du politique, et révélent l'amalgame entre systémes de gestion d;intérêts personnels et systéme de gestion administratif. Les logiques concurrentes, "competing rationales", à l'œuvre lors de la mise en place des réformes, empruntent tant au domaine politique, social, moral, individuel...et conduisent à une négociation constante et à une imparfaite mise en place des réformes.
Si les liens entre réformes politiques et développement infrastructurel peuvent être tissés à l'échelle macroscopique, les questions d'accés à l'eau, de négociations de cet accés dans la ville, dans ces quartiers et entre ces habitants, n'est que bien peu présente dans cette étude. L'approche spécifique par l'institution gouvernementale éclaire davantage les pratiques à l'échelle centrale et au sein de l'institution d'état sans permettre, dans le cadre de ce travail, une descente à l'intérieur des systémes de distribution d'eau, donc de comprendre les stratégies pratiquées par les habitants pour assurer leur accés à la ressource ou la négociation réelle des réseaux d'eau au-delà des réformes néolibérales. La description et l'analyse des types d'accés, de distribution...de l'eau à l'échelle micro-locale manquera surement aux lecteurs qui cherchent ici à "voir" la ville de Khartoum à travers son systéme d'approvisionnement en eau. L'approche institutionnelle prend, dans cet ouvrage, le pas au contraire des monographies habituelles sur les services urbains dans les villes africaines. Ce biais scalaire tient néanmoins davantage des limites et des choix impartis à un travail de recherche, balisé par des exigences académiques et un programme de recherche plus vaste dans lequel plusieurs opus se complétent.

En somme, cet ouvrage donne des clés de lecture aisées pour un lectorat intéressé à comprendre en quoi la crise de l'eau est en réalité une crise de la gestion de l'eau dans l'aire néolibérale contemporaine. L'auteur ouvre également certaines portes pour comprendre le positionnement de la political ecology, également pour des lecteurs peu familier de ce courant. Le complément apporté par l'approche sociologique au sein de l'institution gouvernementale permet une descente au cœur du systéme et replace les individus comme acteurs importants de processus socio-économiques plus larges. Cette entrée par les pratiques sociales dans la mise en place des politiques néolibérales pour la gestion de l'eau à Khartoum et au Soudan est ici novatrice au sein de la géographie humaine. Enfin, la rigueur méthodologique et rédactionnelle de ce travail fait preuve d'exemplarité pour tout exercice académique de ce style. 